\title{
Estudo espaço-temporal do suicídio: uma pesquisa bibliográfica sobre técnicas
}

\author{
Sheila G. Dada ${ }^{1}$, Mônica A. Rodrigues ${ }^{2}$, Etienne A. C. Junior ${ }^{1}$, Ligia V. Barrozo ${ }^{3}$, \\ Marco T. Scanavino ${ }^{2}$, Paulo H. N. Saldiva ${ }^{2}$, Antonio M. Saraiva ${ }^{1}$ \\ ${ }^{1}$ Departamento de Engenharia de Computação e Sistemas Digitais \\ Escola Politécnica da Universidade de São Paulo (EPUSP) - São Paulo, SP - Brasil \\ ${ }^{2}$ Departamento de Psiquiatria e Patologia \\ Faculdade de Medicina da USP (FMUSP) - São Paulo, SP - Brasil \\ ${ }^{3}$ Departamento de Geografia \\ Faculdade de Filosofia, Letras e Ciências Humanas da USP - São Paulo, SP - Brasil \\ \{sheila.dada, monica.rodrigues, cartolano, lija, scanavino, \\ pepino, saraiva\}@usp.br
}

\begin{abstract}
Close to 800,000 people commit suicide worldwide each year, that is one person every 40 seconds. Identifying subpopulations at risk is important for developing preventive strategies. In this article, we present a survey of data science techniques that were implemented in this area. We classify techniques into six phases: data, suicide rate calculation method, descriptive analysis, spatial analysis, temporal analysis, and multivariate analysis. We discuss the various choices of techniques in each of these categories.
\end{abstract}

Resumo. No mundo, aproximadamente 800.000 pessoas se suicidam a cada ano. Para desenvolver planos de prevenção ao ato, é interessante identificar as subpopulações com risco de crescimento da taxa. Neste artigo, apresentase uma revisão bibliográfica sobre as técnicas em ciência de dados abordadas sobre o tema. A abordagem foi dividida em seis etapas: definição dos dados, método de cálculo da taxa de suicídio, análise descritiva, análise espacial, análise temporal e análise multivariada. Em cada etapa, são apresentadas as técnicas utilizadas.

\section{Introdução e objetivo}

No mundo, aproximadamente 800.000 pessoas se suicidam a cada ano. Muitos suicídios ocorrem impulsivamente em momentos de crise e seus fatores de risco são conhecidos. [World Health Organization 2020]. Para desenvolver planos de prevenção ao suicídio é interessante identificar subpopulações com risco de crescimento da taxa [Jaen-Varas et al. 2019]. O estudo espaço-temporal do suicídio auxilia na identificação desses grupos.

Existem diversas abordagens técnicas para estudos espaço-temporais [Atluri, Karpatne e Kumar 2018]. Nos estudos espaço-temporais do suicídio, falta uma análise descritiva mais específica acerca das etapas do estudo e técnicas utilizadas. Portanto, o objetivo deste trabalho é, por meio de uma pesquisa bibliográfica dos estudos espaçotemporais do suicídio, destacar e organizar as etapas e técnicas utilizadas. Dada e Saraiva 2020 enunciam os elementos metodológicos da pesquisa bibliográfica realizada. 


\section{Etapas do Estudo Espaço-Temporal}

Na Tabela 1 é apresentada uma proposta de etapas a se adotar nos estudos e a técnica que geralmente é mais recomendada. Os subitens a seguir detalham cada etapa.

Tabela 1. Etapas de um estudo espaço-temporal do suicídio

\begin{tabular}{|l|l|}
\hline Etapa & Técnica/Abordagem mais abordada \\
\hline 01 - Definição dos dados & Utilização de bases públicas do país \\
\hline 02 - Cálculo da taxa de suicídio & Suavização espacial segregada por sexo e idade \\
\hline $03-$ Análise descritiva & Não há convergência de abordagens \\
\hline 04 - Análise espacial & Agrupamento espacial proposto por Kulldorff \\
\hline $05-$ Análise temporal & Agrupamento espaço-temporal por Kulldorff e Regressão de Joinpoint \\
\hline 06 - Análise multivariada & Técnicas de regressão \\
\hline
\end{tabular}

\subsection{Definição dos Dados}

Além dos dados geográficos e temporais, normalmente os autores também buscam correlacionar o suicídio com outros fatores de risco. Os fatores de risco do suicídio são agrupados em quatro temas: (1) riscos relacionados às comunidades e aos relacionamentos, (2) riscos sociodemográficos, (3) riscos relacionados ao nível individual e (4) riscos ambientais [Jaen-Varas et al. 2019].

Como fonte de informações para estes fatores de risco, os autores utilizam as bases públicas dos órgãos de saúde local, informações do censo, dados públicos de criminalidade (quando aplicável para aquele país/região), e dados públicos ambientais de órgãos governamentais sobre o assunto. Dada e Saraiva (2020) detalham esses dados.

\subsection{Método do Cálculo da Taxa de Suicídio}

Há autores que realizam o cálculo simples da taxa de suicídio: número de mortes/total da população da região. Outro método de cálculo consiste em ajustar a taxa calculada por idade e sexo (cálculo padronizado) [De Arruda et al. 2021] [Santos, Barbosa e Severo 2020] [Bando et al. 2012] [Saman et al. 2012] [Sy et al. 2019].

O suicídio é considerado um evento raro, assim o número de eventos ocorridos pode não ser estatisticamente significativo, o que pode gerar uma taxa com alta variância espacial, devido a flutuações nos tamanhos das populações. Para mitigar essa instabilidade, há pesquisadores que realizam a suavização espacial feita por modelos bayesianos na taxa de suicídio [Fontanella et al. 2018] [Yamaoka et al. 2020].

Com o objetivo de retirar o viés da base e mitigar a instabilidade da taxa de suicídio, recomenda-se a utilização da suavização espacial segregada por sexo e idade.

\subsection{Análise Descritiva}

Objetivando verificar a qualidade dos dados e analisar previamente a correlação das variáveis independentes com o suicídio, realizam-se diversas técnicas na análise descritiva e não há uma abordagem usualmente mais utilizada [Dada e Saraiva 2020]. Portanto, não há uma recomendação única da abordagem técnica a ser seguida.

\subsection{Análise Espacial}

Quanto à análise espacial, o objetivo é entender se há padrão espacial do fenômeno, ou seja, se há tendência a formar agrupamentos espaciais. Deseja-se entender se há algum 
agrupamento de unidades geográficas em que o fator de risco é maior e qual é esse agrupamento. Isso é realizado através do agrupamento espacial. Foram mapeadas quatro técnicas utilizadas: modelo de Kulldorff (2005) [Bando et al. 2012] [Saman et al. 2012] [Fontanella et al. 2018] [Sy et al. 2019], teste de Moran Local, modelo de varredura espacial flexível de Tango-Takahashi e teste de Tango [Yamaoka et al. 2020].

Há também outras abordagens mais simples, listadas por Dada e Saraiva (2020), porém o modelo proposto por Kulldorff (2005) para análises espaciais é a técnica estatística mais utilizada entre os autores.

\subsection{Análise Temporal}

Em relação à análise temporal, um dos objetivos é entender quais unidades geográficas de estudo apresentam variações significativas da taxa de suicídio.

Bando et al. (2012) e Santos, Barbosa e Severo (2020) utilizam a regressão de Joinpoint que identifica pontos de inflexão nas tendências da taxa de suicídio. De Arruda et al. (2021) utilizam a regressão de Prais-Winsten para analisar tendências.

Saman et al. (2012), Fontanella et al. (2018), Yamaoka et al. (2020) e Sy et al. (2019) utilizam técnicas de agrupamento temporal ou espaço-temporal elaboradas por Kuldorff (2005) para descobrir unidades temporais que apresentam alto fator de risco para o suicídio.

Há também outras abordagens mais simples, listadas por Dada e Saraiva (2020), porém o modelo proposto por Kulldorff (2005) e a Regressão de Joinpoint são as técnicas estatísticas mais utilizadas entre os autores.

\subsection{Análise Multivariada}

Quando padrões espaço-temporais significativos são encontrados, procura-se encontrar associação espaço-temporal entre o suicídio e fatores de risco por meio da análise multivariada.

Técnicas de regressão são comumente utilizadas na análise multivariada. Utilizase desde a regressão logística [Bando et al. 2012] [Fontanella et al. 2018] até a comparação entre diversas técnicas [Yamaoka et al. 2020]. Modelos de regressão espacial têm sido utilizados quando os resíduos do modelo OLS apresentam dependência espacial, o que viola a premissa de independência deste modelo.

Há autores que adotam outras técnicas que também variam de complexidade, como as apresentadas por Sy et al. (2019), Saman et al. (2012), Fontanella et al. (2018), Santos, Barbosa e Severo (2020) e listadas por Dada e Saraiva (2020).

Técnicas de regressão são as mais utilizadas entre os autores, porém há uma diversidade de tipos de regressão e não existe uma abordagem específica que seja mais comum nas pesquisas.

\section{Conclusão e Trabalho Futuro}

Neste artigo, a intenção foi trazer uma visão geral sobre as técnicas utilizadas em estudos espaço-temporais sobre o suicídio. Espera-se que este artigo forneça a base para consulta a respeito: das etapas necessárias em um estudo espaço-temporal e das técnicas existentes em cada etapa. 
Uma oportunidade de trabalho futuro é a comparação, definição das limitações e recomendação das técnicas de acordo com a especificidade de cada cenário de estudo sobre o suicídio. Os autores usualmente não mencionam as limitações das técnicas adotadas, então recomenda-se adotar a metanálise para este estudo [Egger M.; Smith G.D.; Altman D. 2008].

\section{Referências}

Atluri, G.; Karpatne, A.; Kumar, V. (2018) "Spatio-temporal data mining: A survey of problems and methods", ACM Computing Surveys, v. 51, n. 4, p. 1-37.

Bando, D. H. et al. (2012) "Suicide rates and income in São Paulo and Brazil: A temporal and spatial epidemiologic analysis from 1996 to 2008", BMC Psychiatry, v. 12.

De Arruda, V. L. et al. (2021) "Suicídio Em Adultos Jovens Brasileiros: Série Temporal De 1997 a 2019”, Ciência \& Saúde Coletiva, p. 2699-2708.

Dada, S. G. e Saraiva, A. M. (2020) "Estudo espaço-temporal do suicídio nas cidades brasileiras", Exame de Qualificação de Mestrado em Ciências, Escola Politécnica da Universidade de São Paulo, São Paulo.

Egger M.; Smith G.D.; Altman D. (2008) "Systematic reviews in health care: metaanalysis in context", John Wiley \& Sons.

Fontanella, C. A. et al. (2018) "Mapping suicide mortality in Ohio: A spatial epidemiological analysis of suicide clusters and area level correlates", Preventive Medicine, v. 106, p. 177-184.

Jaen-Varas, D. et al. (2019) "The association between adolescent suicide rates and socioeconomic indicators in Brazil: a 10-year retrospective ecological study", Brazilian Journal of Psychiatry.

Kulldorff, M. et al. (2005) "A space-time permutation scan statistic for disease outbreak detection”, PLoS Medicine, v. 2, n. 3, p. 0216-0224.

Saman, D. M. et al. (2012) "Does place of residence affect risk of suicide? a spatial epidemiologic investigation in Kentucky from 1999 to 2008", BMC Public Health, v. 12, n. 1, p. 108.

Santos, E. G. DE O.; Barbosa, I. R.; Severo, A. K. S. (2020) "Space-time analysis of mortality by suicide in the state of Rio Grande do norte, Brazil, in the period from 2000 to 2015", Ciencia e Saude Coletiva, v. 25, n. 2, p. 633-643.

Sy, K. T. L. et al. (2019) "Spatiotemporal clustering of suicides in the US from 1999 to 2016: a spatial epidemiological approach", Social Psychiatry and Psychiatric Epidemiology, v. 54, n. 12, p. 1471-1482.

Who World Health Organization (2020) "Suicide prevention", https://www.who.int/health-topics/suicide. Acesso em: 11 abr. 2020.

Yamaoka, K. et al. (2020) "Spatial clustering of suicide mortality and associated community characteristics in Kanagawa prefecture, Japan, 2011-2017”, BMC Psychiatry, v. 20, n. 1, p. 1-15. 